\title{
An LQG Controller Based on Real System Identification for an Active Hydraulically Interconnected Suspension
}

\author{
Yaohua Guo $\mathbb{D}^{1},{ }^{1}$ Bin Wang $\mathbb{D}{ }^{2}$, Anton Tkachev $\mathbb{D}^{2},{ }^{3}$ and Nong Zhang $\mathbb{D}^{2,3}$ \\ ${ }^{1}$ Research Center of Zhengzhou Yutong Bus Co., Ltd., Zhengzhou, Henan, China \\ ${ }^{2}$ School of Automotive and Transportation Engineering, Hefei University of Technology, Hefei, China \\ ${ }^{3}$ School of Mechanical and Mechatronic Engineering, University of Technology, Sydney, Australia \\ Correspondence should be addressed to Bin Wang; 18987669833@163.com
}

Received 7 October 2020; Revised 4 November 2020; Accepted 11 November 2020; Published 24 November 2020

Academic Editor: Xingling Shao

Copyright ( $\odot 2020$ Yaohua Guo et al. This is an open access article distributed under the Creative Commons Attribution License, which permits unrestricted use, distribution, and reproduction in any medium, provided the original work is properly cited.

Rollover prevention is always one of the research hotspots in vehicle design. Active hydraulically interconnected suspension (HIS) is a promising technology to reduce vehicle body roll angle caused by different driving inputs and road conditions. This paper proposes a novel actuator of the active HIS system. The actuator consists of two cylinders, a ball screw, and only one motor. The actuator proposed can reduce the number of motors needed in the system. Meanwhile, forced vibration identification (FVI) is used to identify the transfer function of a half-car physical model and a Kalman state observer is applied to eliminate the influence of sensor noise. The FVI method can eliminate most model uncertainties and hidden variables. Aggressive and moderate optimal linear quadratic Gaussian (LQG) methods are implemented to control the motion of the vehicle body based on the identified transfer function of the physical model. The performance of an active HIS system with an aggressive and moderate LQG controller is compared with that of a passive HIS system. The effectiveness of the LQG controller is validated by simulation and experimental results. Also, the obtained results show that the stabilization speed of the active HIS system is $20 \%$ faster than that of the passive HIS system and the roll angle can be reduced up to $55 \%$ than that of the passive HIS system.

\section{Introduction}

Recently, with the increase in the speed of vehicles on road due to improved driving conditions, the number of rollover accidents is rising. Therefore, anti-roll performance receives increasing attention in the vehicle design process. Among the measures to reduce roll angles such as active steering, differential braking, and active suspension, the application of active steering and differential braking will interfere with normal driving operation. Hence, it will be more worthy to investigate the benefits brought by applying active suspensions for preventing vehicle rollover accidents from happening [1].

The vehicle suspension system is a system that connects wheels and the vehicle body. A suspension system mainly serves two purposes. The first purpose is to isolate the shock and vibration generated by the road excitation to protect occupants from ride discomfort. The second purpose is to keep the wheels in contact with the road surface to improve the stability and safety of the vehicle [2]. Generally, vehicle suspensions can be classified into three types according to energy consumption and control force, i.e., passive, semiactive, and active suspensions $[3,4]$. The passive suspension generally consists of shock absorbers and springs. Parameters of passive suspensions are generally fixed and cannot be adjusted after manufacture. Conflicts are existing between ride comfort and handling performance on passive suspensions $[5,6]$. The semiactive suspension is improved from passive suspension, but the external energy input into the suspension system is insufficient to stabilize the motion of the vehicle body. The active suspension is a kind of suspension which can provide sufficient energy to stabilize the motion of the vehicle body [7]. Compared with passive and semiactive suspension, the active suspension usually realizes its function by four independently acting force actuators. The purpose of the actuators is to produce or dissipate energy under different road excitations. 
In recent decades, many studies have been performed on the suspensions. Smith et al. proposed a passive HIS, and the experimental results show that the roll angle of the proposed passive HIS system is $52.2 \%$ lower than that of passive suspension [8]. The structure and working principle of the passive HIS system are introduced in [9]. There are damper valves, accumulators, fluid circuits, and hydraulic cylinders. The HIS system is designed in a way where the components of the passive suspension system are coupled hydraulically via single- or double-acting cylinders. This external linkage provides extra support to the suspension components at the wheel. The passive HIS system distributes fluid flow to dampers and accumulators in a way that depends on the particular suspension mode in operation. The suspension mode includes bounce, roll, pitch, and warp mode. However, the roll angle of the passive HIS system cannot be reduced to zero because there is no external power to stabilize the vehicle body. Yao et al. proposed a novel dual-mode interconnected suspension with a switching configuration depending on the vehicle working conditions [10]. However, the dual-mode interconnected suspension cannot adjust the posture of the vehicle body actively to counter the roll moment. By comparison, many studies claim that better anti-roll performance can be achieved by the active suspension [11-13]. Wu et al. compared the anti-roll performance of active HIS with passive suspension. The simulation result shows that the roll angle of the active HIS can be reduced to zero degrees while the roll angle of the passive suspension is 6.9 degrees. The load transfer rate of active HIS is $15.2 \%$ lower than that of passive suspension. However, the structure of active HIS in [14] is just a theoretical model. Anton proposed a novel active HIS in [15]. The comparison is made between the passive suspension, passive HIS system, and active HIS system. The results show that the maximum roll angle of the active HIS system is $84.13 \%$ lower than that of passive suspension and $70.7 \%$ lower than that of the passive HIS system. However, this paper does not consider the sensor noise, and the experimental test of the proposed active HIS is not performed. Zhang et al. proposed a new spherical simplex UKF (SSUKF) observer based on an electrohydraulic active suspension. Their test results show that the proposed SSUKF observer can significantly depress body motion and decrease mode energy [16]. However, the work in [16] does not consider the model uncertainties which are inevitable in practical application. The model uncertainties widely exist in the real world [17]. Many studies have been dedicated to eliminating the passive influence of the model uncertainties [18-20]. However, the numerical method cannot fully eliminate the model uncertainties due to the complexity of the real physical system. Liang et al. proposed a novel Chebyshev interval method to reduce the influence of the model uncertainties such as pipe friction coefficient and fluid damping coefficient [21].
However, the method is not convenient to implement in real car experiment.

The active suspension proposed in this paper is a rollplane active HIS system with an independent force actuator, which is evolved from the passive HIS system [9]. The objective of this paper is to design an LQG controller with a Kalman state observer based on the proposed roll-plane active HIS system. The control objective of the proposed controller is to minimize the roll angle of the vehicle's body to prevent rollover accidents. The FVI test is used to reduce the influence of model uncertainties.

This paper is organized as follows: in Section 2, the model of the active HIS system for a sport utility vehicle (SUV) is introduced and the state-space equation is derived; in Section 3, the transfer function of the open-loop control system is identified through the FVI test, which makes preparation for the closed-loop control system design; the LQG controller with the Kalman state observer is designed in Section 4; MATLAB simulation and the bench test are performed in Section 5 .

\section{Modeling}

Passive HIS systems have been modeled and thoroughly studied in [9]. The studies proved that an HIS system has a promising potential for the improvement of driving safety and rollover prevention of vehicles. Besides, these studies also provide opportunities to further improve the HIS system. The proposed active HIS system in this paper is developed based on a passive HIS system. The mechanism of the proposed active HIS system is shown in Figure 1, in which an actuator is added in the passive HIS system. The active HIS system consists of an actuator formed by a cylinder-piston unit and its driving motor. As the piston moves, the hydraulic fluid flows from one side to another creating pressure difference between hydraulic circuits P1 and P2. The differential pressure is then converted into an additional anti-roll force exerted on the vehicle body. The novel system proposed in this paper does not need an additional accumulator for hydraulic fluid. Neither does this system need four active actuators in [22]. This novel model proposed in this paper reduces the number of actuators, accumulators, and pumps needed in the system while realizing the same function. The proposed actuator reduces significant costs for the development of an active suspension system.

A half-car model equipped with an active HIS system is shown in Figure 2. The state-space equations of the rollplane half-car model can be obtained by applying the Lagrange method. The detailed derivation process is shown in [15]. The state-space equations are given in equations (1)-(5), and the state-space variables are given in Table 1: 


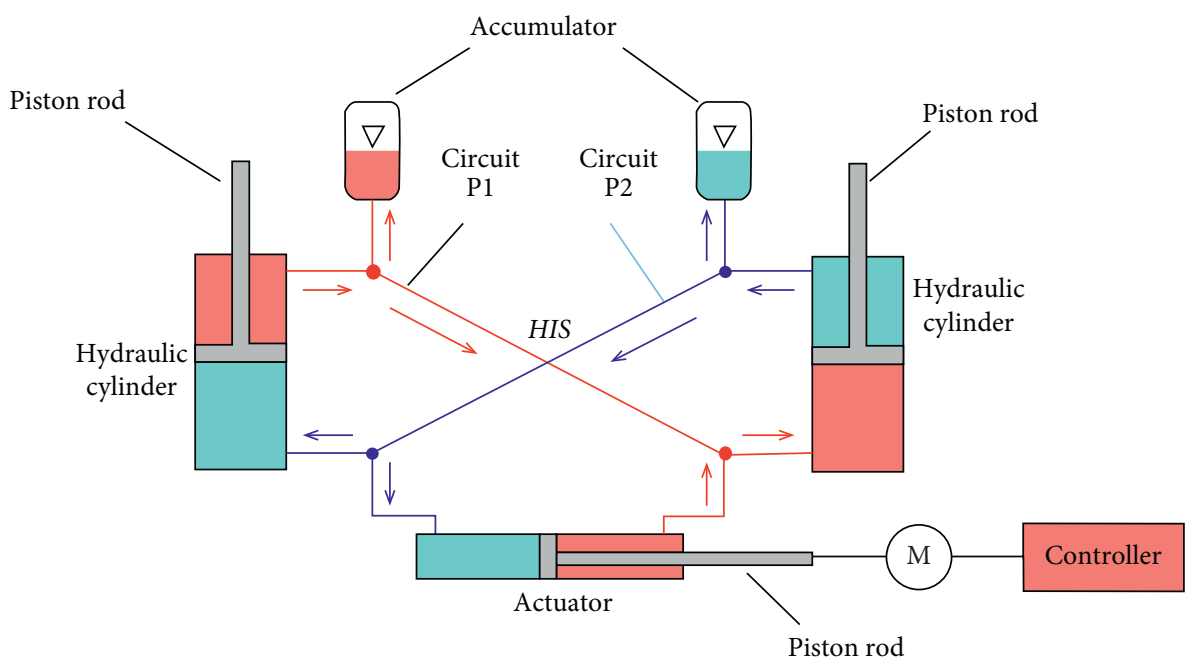

FiguRE 1: Mechanism of the proposed active HIS system.

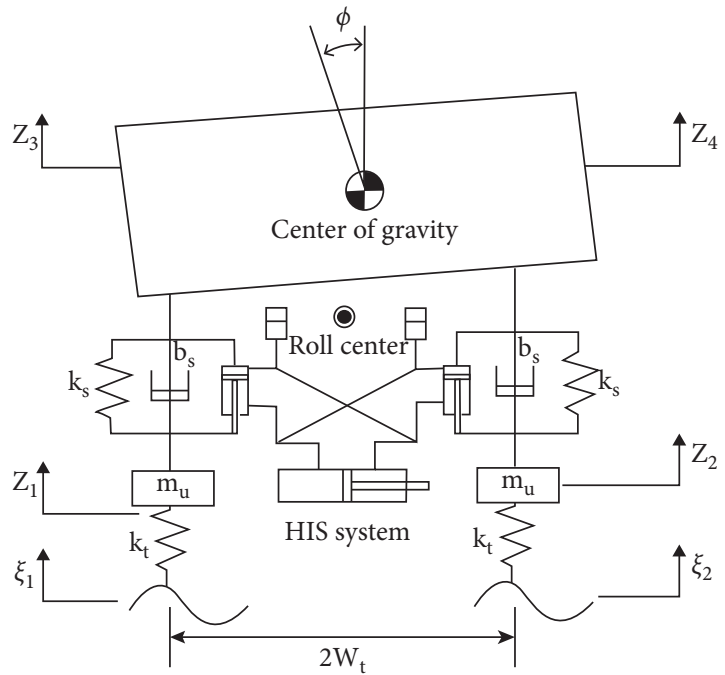

FIgURE 2: Half-car model with the active HIS system.

TABLE 1: State-space model variables.

\begin{tabular}{lcc}
\hline Variable & Symbol & Unit \\
\hline State variable $\left(x=x_{1}, x_{2}, x_{3}, x_{4}, x_{1}, x_{2}, x_{3}, x_{4}\right)^{T}$ & & \\
Left wheel vertical displacement & $x_{1}$ & $\mathrm{~m}$ \\
Right wheel vertical displacement & $x_{2}$ & $\mathrm{~m}$ \\
Vehicle body vertical displacement on the left & $x_{3}$ & $\mathrm{~m}$ \\
Vehicle body vertical displacement on the right & $x_{4}$ & $\mathrm{~m}$ \\
Input variables $\left(u=\omega_{1}, \omega_{2}, f_{r}, \Delta x_{a}\right)^{T}$ & & \\
Left road input disturbance & $\omega_{1}$ & $\mathrm{~m}$ \\
Right road input disturbance & $\omega_{2}$ & $\mathrm{~m}$ \\
Equivalent roll force due to lateral acceleration & $f_{r}$ & $\mathrm{~N}$ \\
Actuator displacement & $\Delta x_{a}$ & $\mathrm{~m}$ \\
Output variable $y=\left(\Delta x_{1}, \Delta x_{2}, x_{s}, \varphi_{s}\right)^{T}$ & & \\
Left suspension deflection & $\Delta x_{1}$ & $\mathrm{~m}$ \\
Right suspension deflection & $\Delta x_{2}$ & $\mathrm{~m}$ \\
Vehicle center of gravity vertical displacement & $x_{s}$ & $\mathrm{~m}$ \\
Vehicle body roll angle & $\varphi_{s}$ & $\mathrm{rad}$ \\
\hline
\end{tabular}

$$
\begin{aligned}
\dot{x} & =A x+B u+G \xi, \\
y & =C x, \\
A & =\left[\begin{array}{cc}
{[0]_{4 \times 4}} & {[I]_{4 \times 4}} \\
-\widehat{M}^{-1}(\widehat{K}) & -\widehat{M}^{-1}(\widehat{D})
\end{array}\right], \\
B & =\widehat{M}^{-1} K_{h a},
\end{aligned}
$$$$
\left.C=\left[\begin{array}{cccc}
-1 & 0 & 1 & 0 \\
0 & -1 & 0 & 1 \\
0 & 0 & \frac{1}{2} & \frac{1}{2} \\
0 & 0 & -\frac{1}{2 W_{t}} & \frac{1}{2 W_{t}}
\end{array}\right][0]_{4 \times 4}\right] \text {, }
$$

\section{System Identification}

3.1. Model Base Control through System Identification. According to modern control theory, feedback controllers of mechanical systems can be classified into two major types, i.e., the model-dependent type and model-independent type. Model-dependent controllers, such as state feedback controllers, necessarily require preceding knowledge of transfer function or state-space information of the real physical system. The other type is the model-independent type which does not need preceding knowledge of the physical system. The proportional integral derivative (PID) controller is one of the typical model-independent controllers which can be tuned by trial and error conveniently. However, the PID 


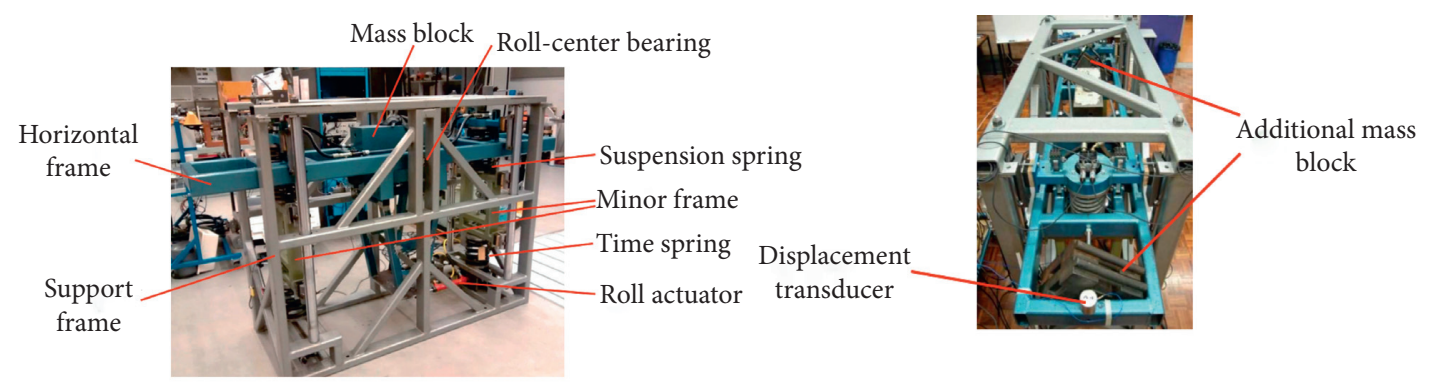

(a)

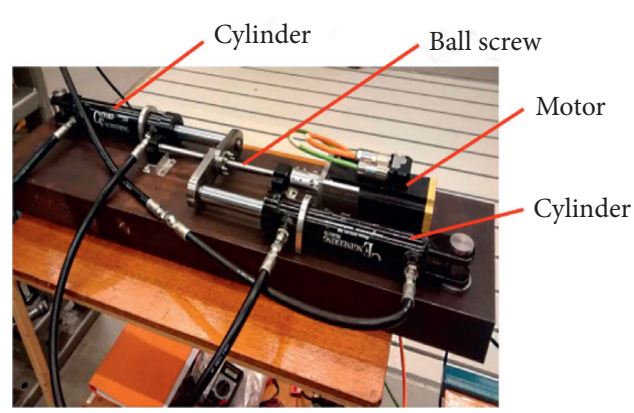

(c) (b)

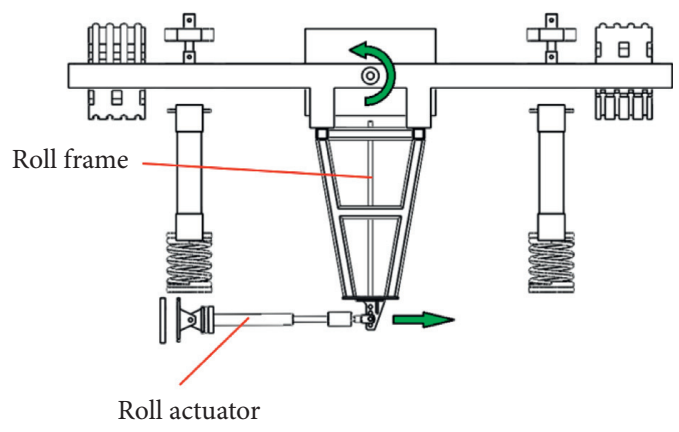

(d)

Figure 3: Half-car testing rig. (a) The main view of the test stand. (b) The top view of the test stand. (c) The proposed actuator of the active HIS system. (d) Schematic diagram showing the translating method of roll moment to roll angle of the main body.

controller is sensitive to noise and fails to provide acceptable performance under transducer noise. Based on these characteristics, the model-dependent controller is chosen in this paper. Therefore, the accurate identification of the transfer function of the physical system is essential for the model-dependent controller design.

3.2. Identification Experiment of Real Systems. Although many numerical derivation methods of the transfer function and state-space equations have been proposed, they cannot fully reflect the properties of the real physical systems due to hidden variables and model uncertainties. In this paper, the powerful FVI experiment is chosen to identify the transfer function and state-space equations of a real physical system. The acquired test results from the FVI experiment will fully reflect the property of the real system including hidden variables and model uncertainties. The test rig of the half-car model equipped with an active HIS system is shown in Figure 3.

The test rig is built upon a support frame acting as a support. The horizontal frame is suspended on two suspension springs located on the left and the right side, respectively. Two additional mass blocks placed on the horizontal frame are used to increase the inertia of the system. The horizontal frame can only move vertically and rotate around the bearing axis. The purpose of the horizontal frame is to simulate the vehicle body. The mass block on the horizontal frame is to simulate the inertia properties of a real car. There are two minor frames on both sides that represent the unsprung mass including wheels and tires. The functionality of the roll actuator is to push and pull the roll frame against the roll-center bearing. The roll frame is attached to the bottom of the horizontal frame. The active HIS system is embedded into the half-car assembly.

The external disturbance is exerted on the test rig by pushing the roll actuator against the roll-center bearing. The horizontal frame representing the vehicle body will incline in response to this external input. The vertical displacement of the horizontal frame is measured by linear variable displacement transducers (LVDTs) in the left and right side of the horizontal frame, respectively. The measured data from LVDT will be sent to the controller to analyze the roll angle of the horizontal frame. With the signal from the controller, the motor will drive the actuator to oppose the roll moment disturbance and decrease the roll angle value.

The schematic diagram of the identification experiment is shown in Figure 4. According to the theory of secondorder systems, when subjecting the half-car test rig equipped with an active HIS system to a specific sine wave excitation, the response will be a sine wave with the same frequency but with different amplitude and phase. By taking the fast Fourier transform of the input disturbance and output of the test rig, the response magnitude ratio and phase angle difference between the input and output can be obtained. After each test, a data point of the Bode diagram can be added. The identification experiment is performed at one particular frequency each time. The test should be carried out enough times to fully cover the range of frequency of interest. In this paper, the frequency range of interest is $0.1 \sim 10 \mathrm{~Hz}$. Based on the data points in $0.1 \sim 10 \mathrm{~Hz}$, the identified curve can be fitted as Figure 5. The transfer function identified is as follows: 


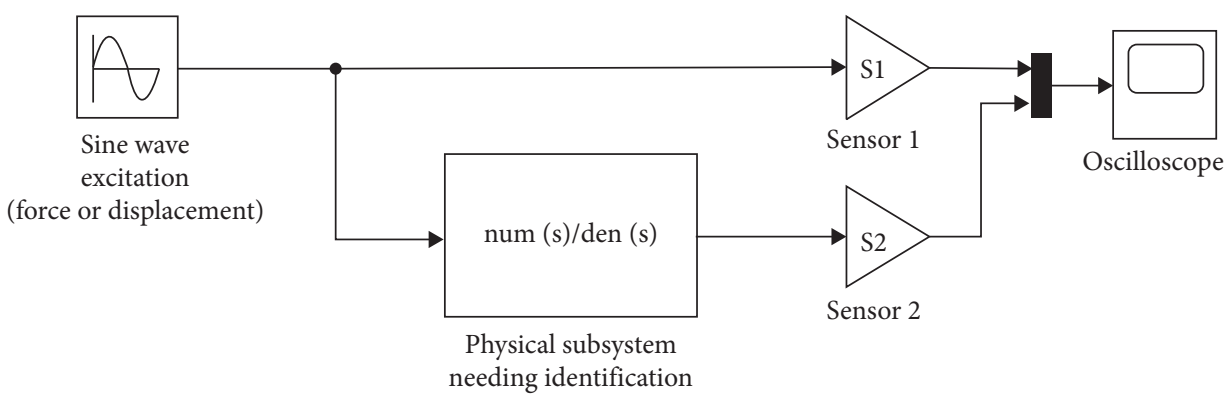

FIgURE 4: Schematic diagram of system identification experiment.
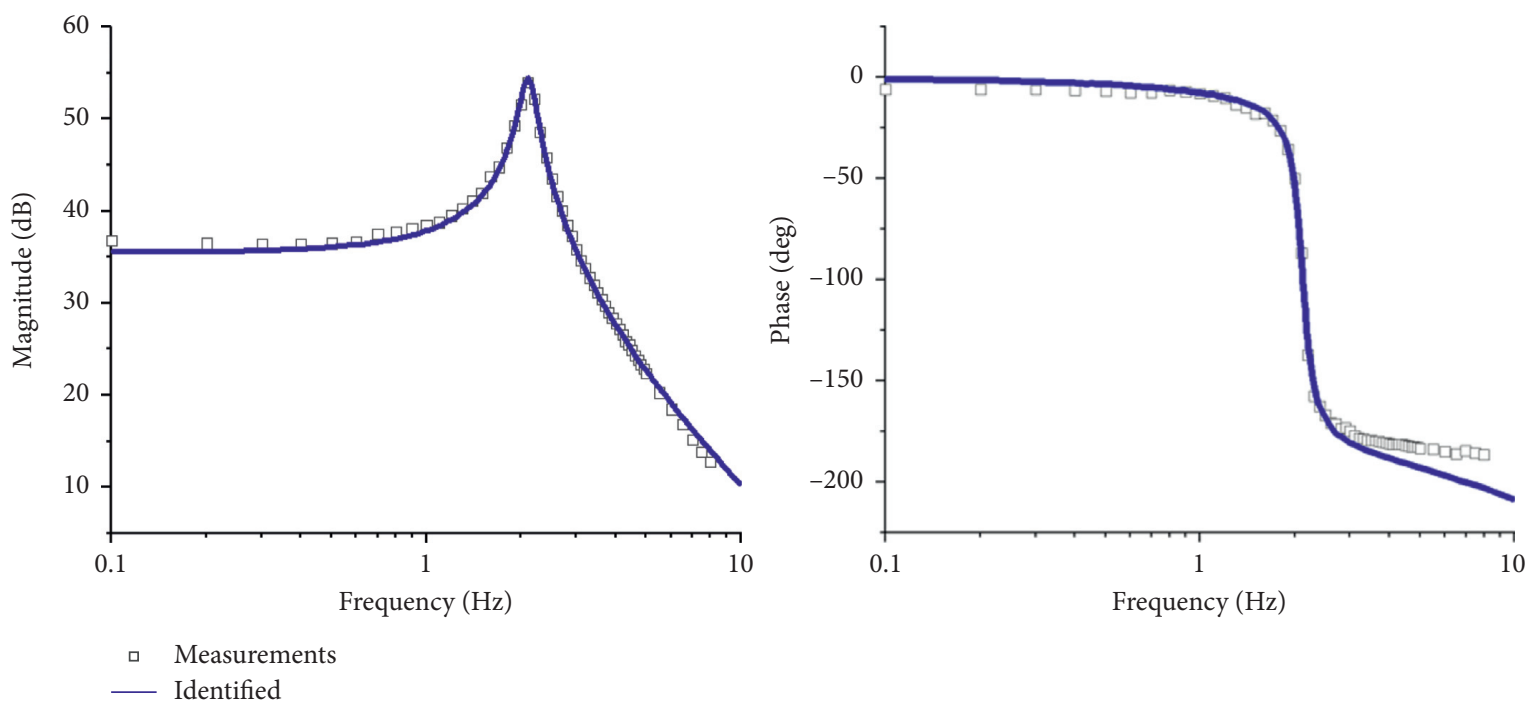

Figure 5: Experimental identification of the open-loop system from roll moment disturbance to roll angle.

$$
T_{0}=\frac{-97.64 s+10630}{s^{2}+1.547 s+176.7}
$$

\section{Control System Design and Validation}

4.1. Control System Design. Based on the transfer function identified from the identification experiment, the controller can be accurately designed. To suppress the motion of the vehicle body, a linear quadratic regulator (LQR) controller is chosen for the active HIS system. LVDTs are used in this paper to measure the vertical displacement of wheels and the vehicle body. However, the electromagnetic noise of LVDT will affect the function of the system, so the Kalman state observer method is used in this paper to filter the noise. When LQR and Kalman observers are working together, they are equivalent to an LQG controller which is widely used in many industries for its high reliability and robustness.

The control system of the active HIS system is illustrated in Figure 6. The displacement data measured by the left and right LVDTs are converted into roll angle firstly, and then roll angle data will be processed by the Kalman state observer to reduce the influence of sensor noise. The corrected roll angle data will be sent to the LQR controller to adjust the rotation speed of the actuator motor, which will move the actuator to produce an anti-roll torque.

In the process of LQR controller design, two different controller settings are chosen: the moderate and aggressive settings. For the moderate setting, $Q_{\mathrm{m}}$ and $G_{\mathrm{m}}$ are defined in equations (7) and (8). For the aggressive setting, $Q_{\mathrm{a}}$ and $G_{\mathrm{a}}$ are defined in equations (9) and (10). In this paper, other relevant parameters of the LQR controller are derived in MATLAB:

$$
\begin{aligned}
Q_{m} & =\left[\begin{array}{cccc}
1 & 0 & 0 & 0 \\
0 & 1 & 0 & 0 \\
0 & 0 & 10^{3} & 0 \\
0 & 0 & 0 & 10^{5}
\end{array}\right], \\
G_{m} & =\left[\begin{array}{llll}
11.29 & 26.17 & 31.95 & 316.22
\end{array}\right], \\
Q_{a} & =\left[\begin{array}{cccc}
1 & 0 & 0 & 0 \\
0 & 1 & 0 & 0 \\
0 & 0 & 10^{3} & 0 \\
0 & 0 & 0 & 10^{7}
\end{array}\right], \\
G_{a} & =\left[\begin{array}{llll}
23.72 & 91.81 & 191.43 & 3162.27
\end{array}\right] .
\end{aligned}
$$




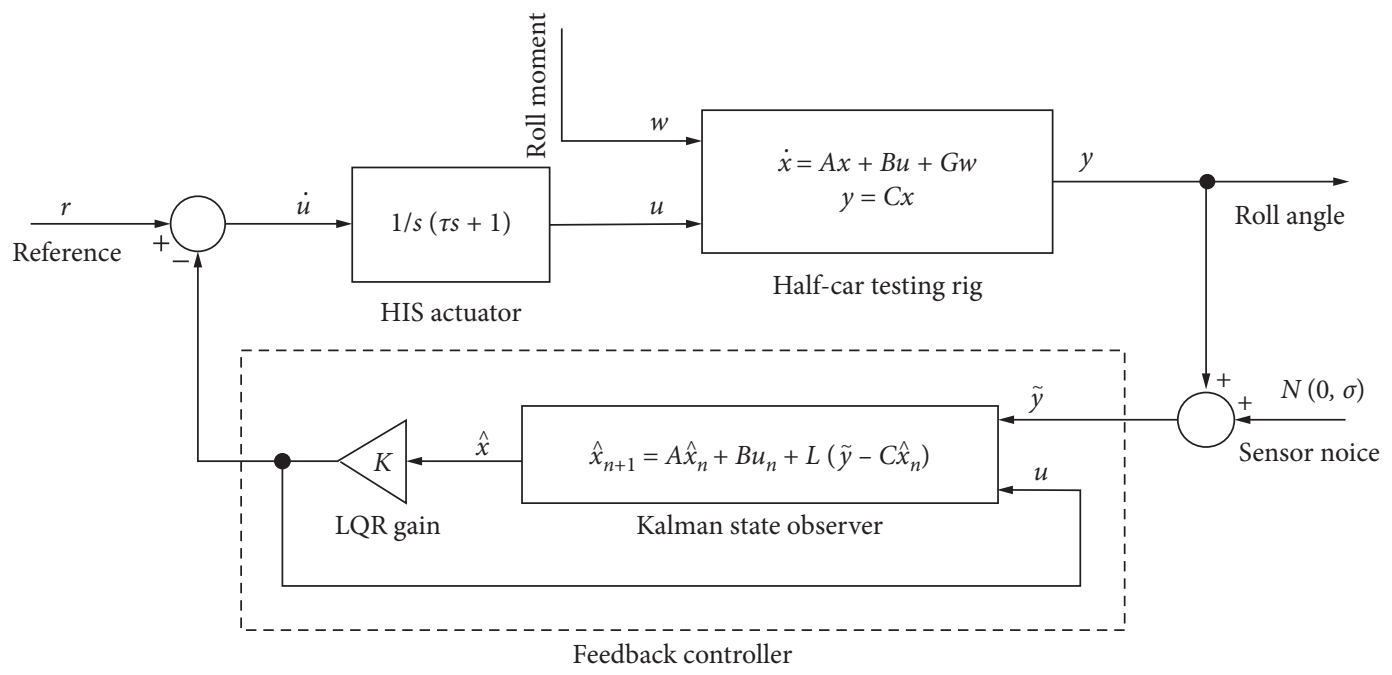

Figure 6: Closed-loop control system.
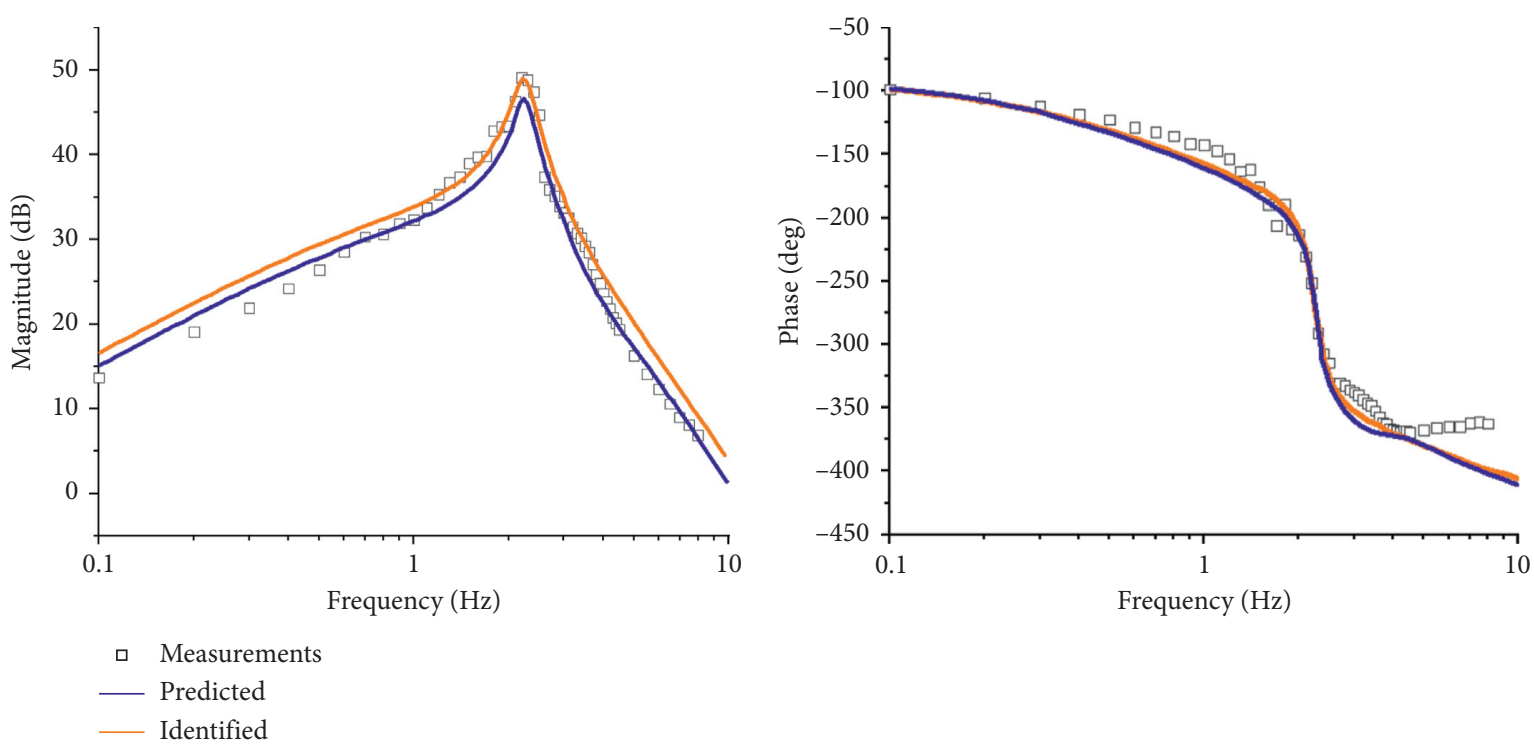

FIGURE 7: Experimental identification of the closed-loop system from disturbance to roll angle when controlled with an LQG controller at a moderate setting.

4.2. Control System Performance. The accuracy of the FVI method is verified by comparing the predicted curve with the identified curve in the Bode diagram. The identified curve is fitted by measurement data of the real physical system. The predicted curve is converted from the state-space equation deduced in [15]. The comparison result is shown in Figures 7 and 8 .

The identified transfer function of the test rig from disturbance to roll angle when controlled with LQG at a moderate setting is shown as follows:

$$
T_{m}=\frac{-472000 s+8555}{s^{4}+55.65 s^{3}+540.9 s^{2}+11140 s+43880} \text {. }
$$

The identified transfer function of the test rig from disturbance to roll angle when controlled with LQG at an aggressive setting is shown as follows:

$$
T_{a}=\frac{2530000 s-189900}{s^{4}+148.6 s^{3}+9387 s^{2}+49050 s+1586000} .
$$

From Figures 7 and 8, it can be concluded that the identified curve and the predicted curve are in good agreement. The slight difference between the identified curve and predicted curve is caused by model uncertainties and hidden variables, which once again validates the accuracy of the FVI method.

To verify the performance of the control system, the Bode diagram of the open-loop system, closed-loop system with the moderate setting, and closed-loop system with the aggressive setting is compared, and the result is shown in Figure 9.

As seen in Figure 9, LQG controllers effectively attenuate the magnitude of response in the low-frequency domain. 

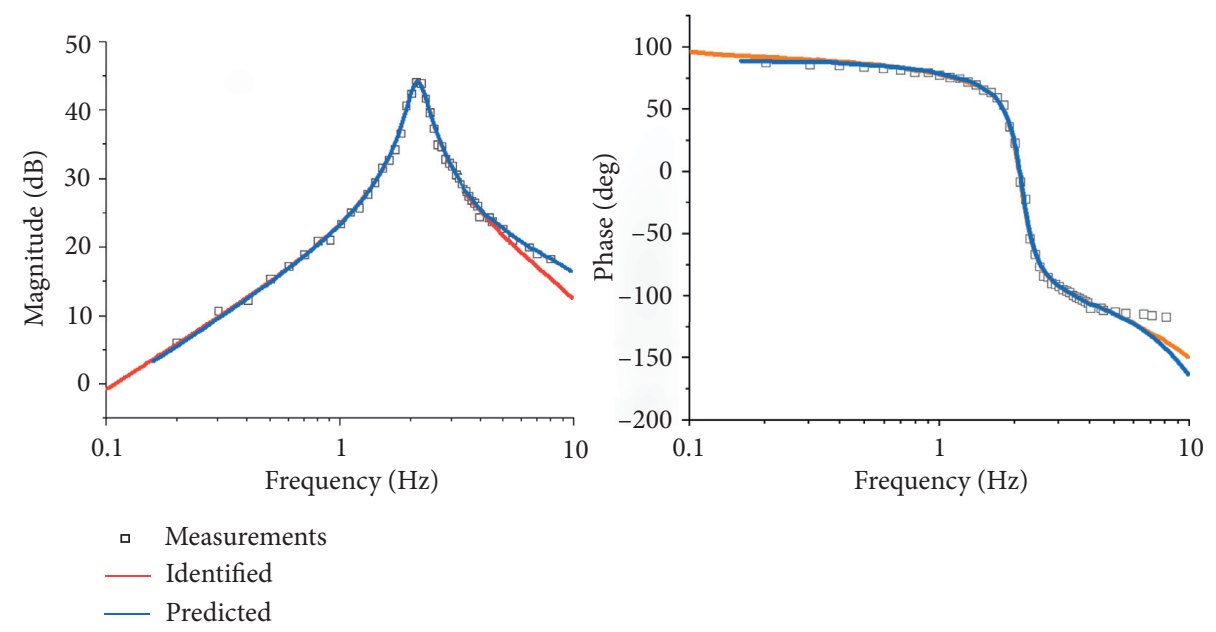

FIGURE 8: Experimental identification of the closed-loop system from roll moment disturbance to roll angle when controlled with an LQG controller at aggressive setting.

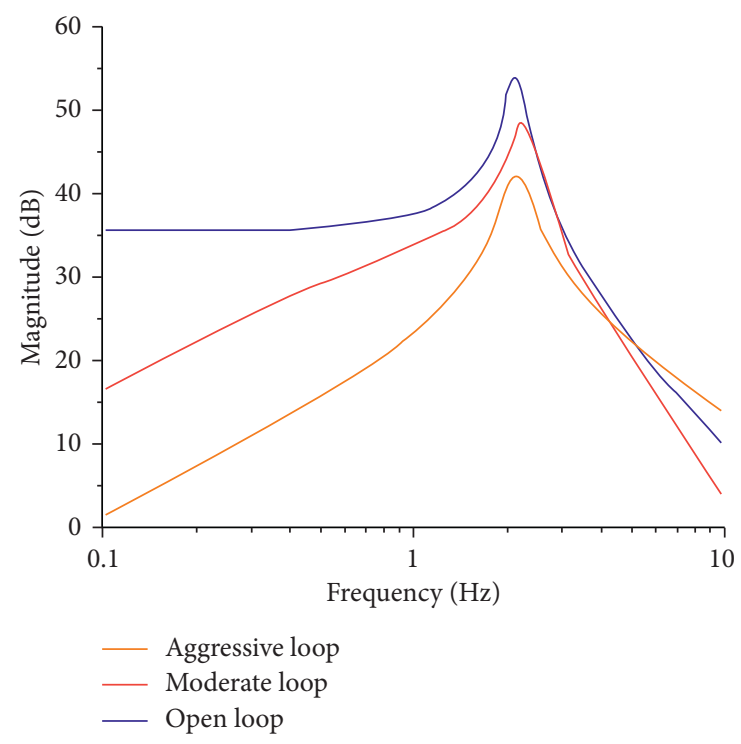

Figure 9: Comparison between controlled and uncontrolled models.

The frequency of the peak points is approximately $2.3 \mathrm{~Hz}$. The peak value of the active HIS system with aggressive LQG is $21.9 \%$ lower than that of the passive HIS system. The peak value of the active HIS system with aggressive LQG is $10.1 \%$ lower than that of the passive HIS system.

\section{Simulation and Bench Test}

5.1. Simulation. The pole map of the open-loop and the closed-loop systems is displayed in Figure 10.

As seen in Figure 10, the poles of the closed-loop system with the LQG controllers are located further to the left than the open-loop system, which validates the excellent performance of the designed LQG controllers. Besides, the LQG controller with an aggressive setting shows better stability performance than the controller with a moderate setting. The simulation result of the response of the open-loop and closedloop systems under step excitation is shown in Figure 11.

It can be seen from Figure 11 that the open-loop system has the highest response amplitude and the closed-loop system with an aggressive setting controller has the lowest response amplitude. After stabilization, the half-car body with an open-loop system still tilts due to the influence of step excitation. However, the response amplitude of the closed-loop system after stabilization is zero. It is because that the controller will detect roll angle information and adjust the vehicle body position automatically. The oscillation stop time of the system with an aggressive and moderate controller is approximately the same, and both are lower than that of the open-loop system. 


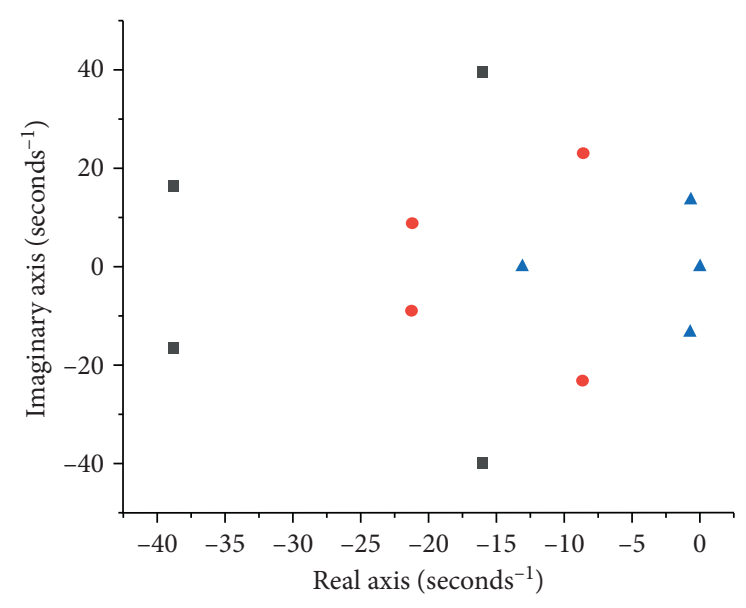

A Aggressive LQR

- Moderate LQR

- Original system

FIGURE 10: Expected pole map of the open-loop and closed-loop systems.

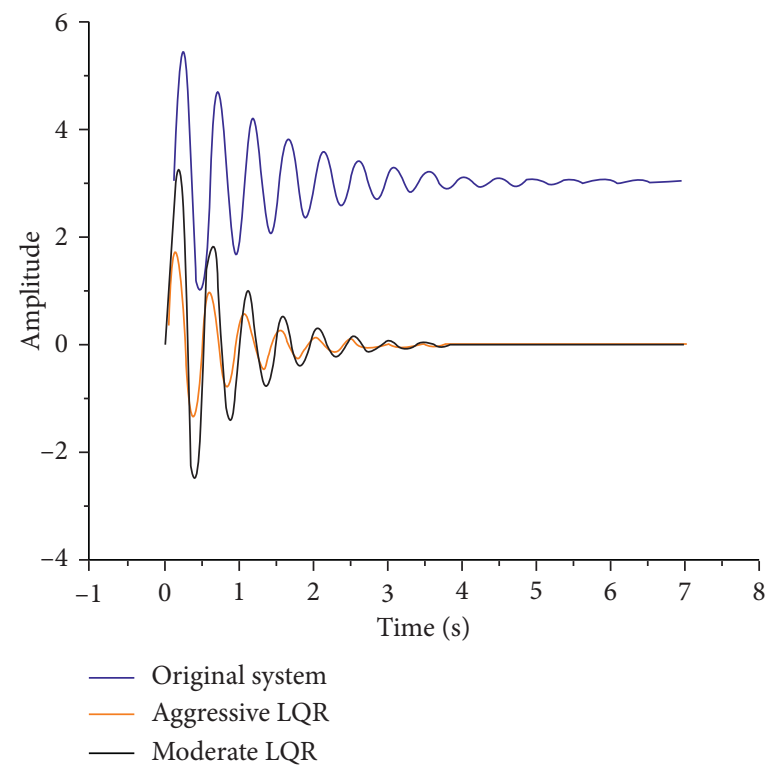

FIGURE 11: Simulation result of the response of the open-loop and closed-loop systems under step excitation.

5.2. Drop Test. The purpose of the drop test is to imitate the sharp steering which is defined in J-turn or Fishhook maneuver by the National Highway Traffic Safety Administration (NHTSA). The test method is used to lift one side of the horizontal frame with a crane and then drop it suddenly. The readings of the roll angle and hydraulic pressure are recorded in the hydraulic circuit. The test result is shown in Figure 12.

Compared with Figure 11, the simulation result and drop test result are in good agreement, which demonstrates that the identified model accurately characterizes the real physical system. The stabilization time of the passive HIS system is $5 \mathrm{~s}$, while the time for the active HIS system is $4 \mathrm{~s}$. The stabilization speed of the active HIS system is $20 \%$ faster than that of the passive HIS system. The maximum roll angle for the passive HIS system is 4.56 degrees while that for the active HIS system with moderate LQG and aggressive LQG is 3.0 degrees and 2.05 degrees, respectively. In other words, the roll angle of the active HIS system can be reduced by up to $55 \%$ than that of the passive HIS system. Besides, the active HIS system with aggressive LQG will consume more energy though the roll angle can be reduced further. The choice of aggressive and moderate LQG controller will depend on the tunning of the active HIS system. The hydraulic pressure in the circuit is presented in Figure 13.

As seen in Figure 13, the maximum pressure in the hydraulic circuit of the active HIS system is $42.9 \%$ higher than that in the circuit of the passive HIS system. The pressure difference is generated by the actuator, which demonstrates the positive role of the actuator in suppressing the vehicle body roll motion. 


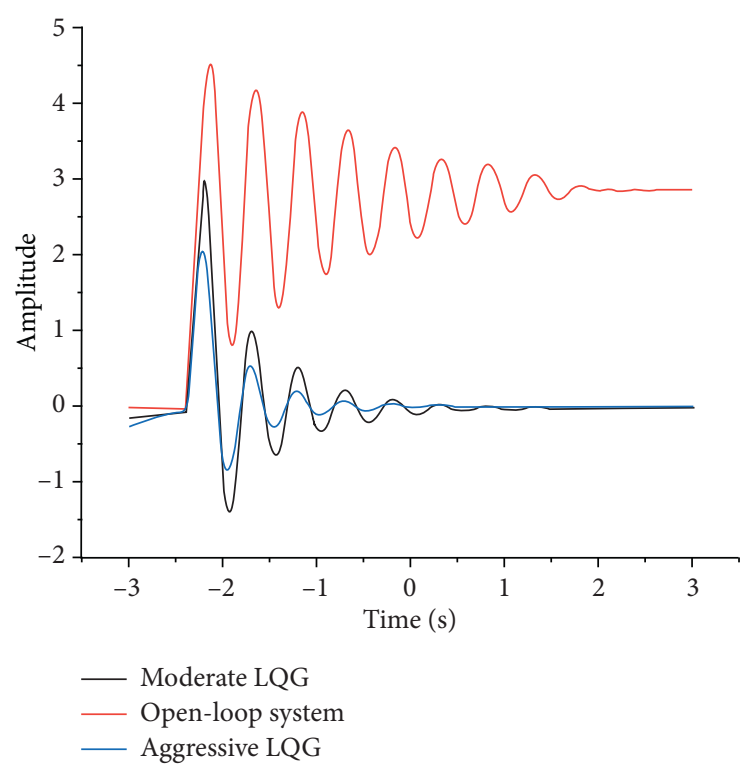

Figure 12: Pressure measurement results in the drop test.

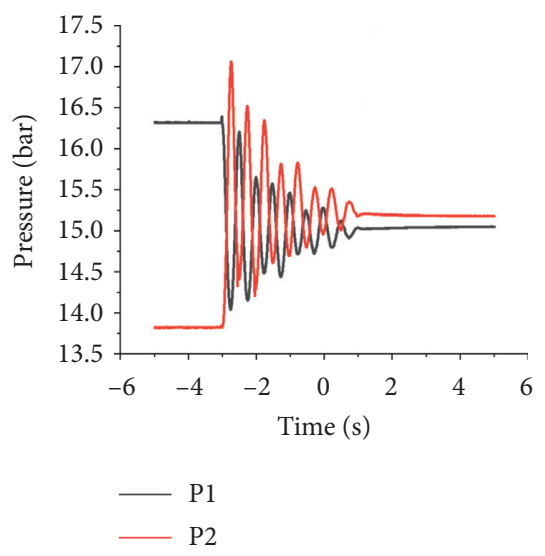

(a)

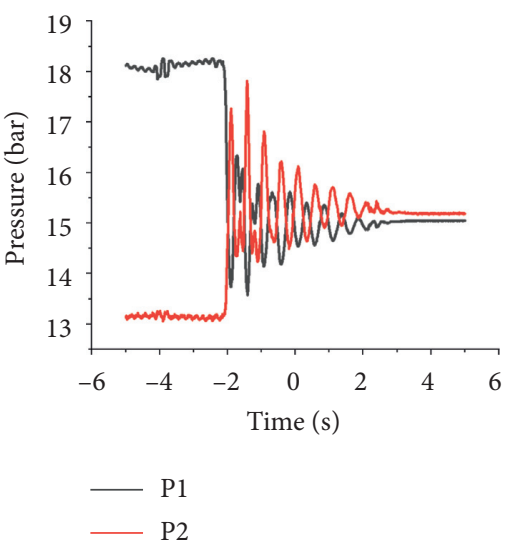

(b)

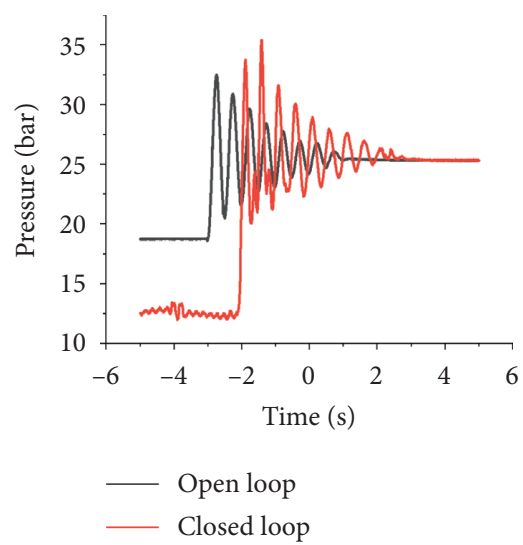

(c)

Figure 13: Pressure measurement results. (a) Open-loop system pressure. (b) Closed-loop system pressure. (c) Differential pressure compared between open- and closed-loop systems.

\section{Conclusions}

This paper firstly proposes a novel active HIS system, which consists of components in the passive HIS system and an actuator formed by a cylinder-piston unit and its driving motor. The new active HIS system saves significant costs by using only one actuator while realizing the same functionality. Secondly, it presented a specific design of the LQG controller and Kalman observer based on the active HIS system. During the controller design process, an FVI method is used in the active suspension development process to identify the transfer function of the real physical system. This method of controller design is more effective as it eliminates most model uncertainties and hidden variables to the maximum extent possible. It makes the controller design process straightforward without the necessity of tunning by trial and error. Moreover, this approach is sure to be consistent with the real system that the controller is designed for. The simulation and bench test results demonstrate the excellent performance of the proposed control method and system identification method. The bench test results show that the stabilization speed of the active HIS system is $20 \%$ faster than that of the passive HIS system and the roll angle can be reduced up to $55 \%$ than the passive HIS system. The proposed active HIS system can be applied to the real car to reduce the overall cost and achieve better anti-roll performance at the same time. The system is potentially useful for heavy vehicles like trucks, buses, and SUVs.

As future work, we would like to design a switchable controller integrated with anti-pitch, anti-roll, and antibounce function based on motion-mode energy detection. The real car status will be detected in real-time to switch the controller to the relative mode. 


\section{Data Availability}

The datasets generated and analyzed in the current study may be obtained from the corresponding author upon reasonable request.

\section{Conflicts of Interest}

The authors declare no conflicts of interest regarding the publication of this paper.

\section{Acknowledgments}

The authors are grateful to the Hefei University of Technology, China, the University of Technology, Sydney, and Zhengzhou Yutong Bus Co., Ltd., China, for their support.

\section{References}

[1] S.-a. Chen, Y.-m. Cai, J. Wang, and M. Yao, "A novel LQG controller of active suspension system for vehicle roll safety," International Journal of Control, Automation and Systems, vol. 16, no. 5, pp. 2203-2213, 2018.

[2] S. Zhu, G. Xu, A. Tkachev, L. Wang, and N. Zhang, "Comparison of the road-holding abilities of a roll-plane hydraulically interconnected suspension system and an anti-roll bar system," Proceedings of the Institution of Mechanical Engineers, Part D: Journal of Automobile Engineering, vol. 231, no. 11, pp. 1540-1557, 2017.

[3] S.-A. Chen, J.-C. Wang, M. Yao, and Y.-B. Kim, "Improved optimal sliding mode control for a non-linear vehicle active suspension system," Journal of Sound and Vibration, vol. 395, pp. 1-25, 2017.

[4] H. E. Tseng and D. Hrovat, "State of the art survey: active and semi-active suspension control," Vehicle System Dynamics, vol. 53, no. 7, pp. 1034-1062, 2015.

[5] Y. Zhang, K. Guo, D. Wang, C. Chen, and X. Li, "Energy conversion mechanism and regenerative potential of vehicle suspensions," Energy, vol. 119, pp. 961-970, 2017.

[6] J.-L. Wu, "A simultaneous mixedLQR/Hoocontrol approach to the design of reliable active suspension controllers," Asian Journal of Control, vol. 19, no. 2, pp. 415-427, 2017.

[7] L.-X. Guo and L.-P. Zhang, "Robust Ho control of active vehicle suspension under non-stationary running," Journal of Sound and Vibration, vol. 331, no. 26, pp. 5824-5837, 2012.

[8] W. Smith, N. Zhang, and W. Hu, "Hydraulically interconnected vehicle suspension: handling performance," $V e$ hicle System Dynamics, vol. 49, no. 1-2, pp. 87-106, 2011.

[9] W. A. Smith, N. Zhang, and J. Jeyakumaran, "Hydraulically interconnected vehicle suspension: theoretical and experimental ride analysis," Vehicle System Dynamics, vol. 48, no. 1, pp. 41-64, 2010.

[10] Q. Yao, X. Zhang, K. Guo, Y. Yang, and J. Feng, "Study on a novel dual-mode interconnected suspension," International Journal of Vehicle Design, vol. 68, no. 1-3, pp. 81-103, 2015.

[11] S. F. Vander Westhuizen and P. S. Els, "Slow active suspension control for rollover prevention," Journal of Terramechanics, vol. 50, no. 1, pp. 29-36, 2013.

[12] S. Yim, "Design of a robust controller for rollover prevention with active suspension and differential braking," Journal of Mechanical Science and Technology, vol. 26, no. 1, pp. 213-222, 2012.
[13] L. Chai and T. Sun, "The design of LQG controller for active suspension based on analytic hierarchy process," Mathematical Problems in Engineering, vol. 2010, Article ID 701951, 19 pages, 2010.

[14] X. Wu, B. Zhou, and G. Wen, "A study on anti-roll control of half-car model active interconnected suspension with consideration of actuator dynamics," Journal of Vibration and Shock, vol. 36, no. 12, pp. 150-154, 2017.

[15] A. A. Tkachev and N. Zhang, "Active hydraulically interconnected suspension. modeling and simulation," SAE Technical Papers, 2017.

[16] J. Zhang, F. Ding, N. Zhang, S. Chen, and B. Zhang, "A new SSUKF observer for sliding mode force tracking $\mathrm{H} \infty$ control of electrohydraulic active suspension," Asian Journal of Control, vol. 22, no. 2, pp. 761-778, 2018.

[17] X. Shao, S. Haonan, and W. Zhang, "Event-triggered neural intelligent control for uncertain nonlinear systems with specified-time guaranteed behaviors," Neural Computing and Applications, 2020.

[18] X. Shao, L. Wang, J. Li, and J. Liu, "High-order ESO based output-feedback dynamic surface control for quadrotors under position constraints and uncertainties," Aerospace Science and Technology, vol. 89, pp. 228-298, 2019.

[19] X. Shao and Y. Shi, "Neural adaptive control for MEMS gyroscope with full-state constraints and quantized input," IEEE Transactions on Industrial Informatics, vol. 16, no. 10, pp. 6444-6454, 2020.

[20] X. Shao, S. Haonan, and W. Zhang, "Fuzzy wavelet neural control with improved prescribed performance for MEMS gyroscope subject to input quantization," Fuzzy Sets Systems, 2020.

[21] J. Liang, J. Wu, N. Zhang et al., "Interval uncertain analysis of active hydraulically interconnected suspension system," Advances in Mechanical Engineering, vol. 8, no. 5, pp. 1-14, 2016.

[22] W. Sun, H. Gao, and B. Yao, "Adaptive robust vibration control of full-car active suspensions with electrohydraulic actuators," IEEE Transactions on Control Systems Technology, vol. 21, no. 6, pp. 2417-2422, 2013. 